\title{
Subclinical hypothyroidism in pregnancy: to treat or not to treat
}

\author{
Ulla Feldt-Rasmussen
}

Received: 18 August 2013/Accepted: 21 August 2013/Published online: 10 October 2013

(C) Springer Science+Business Media New York 2013

The published study [1] aimed to observe the pattern of thyroid function in pregnant women with subclinical hypothyroidism treated with levothyroxine (LT4) throughout pregnancy and to obtain the optimal dosages of LT4 for pregnant women with subclinical hypothyroidism. The authors screened 4,102 pregnant women from 10 hospitals of the Shenyang region, northeast China, and enrolled 89 women among 938 with subclinical hypothyroidism defined by serum thyrotropin $>2.5 \mathrm{mU} / \mathrm{L}$ and "normal" free thyroxine (T4), but ended by including 56 compliant women. They were divided in three groups based on different serum TSH concentrations, given three different dosages of LT4 and followed with 4-week intervals by measuring thyroid function tests until week 36 of gestation. Ten of the patients had to be dose adjusted to remain within target, the others stayed on the same dose.

The authors conclude that different LT4 doses can be chosen according to the baseline TSH levels of pregnant women, and maintain serum TSH levels of $79.3-90 \%$ patients in the ideal range. $20 \%$ need adjustment of therapy during second and third trimester. Careful monitoring of serum TSH and free T4 each month is, therefore, advocated.

The study is thus a small descriptive contribution to demonstrating the biochemical pattern during different LT4 dosages during pregnancy of women with subclinical hypothyroidism. However, the study leaves a lot of caveats and unanswered questions.

U. Feldt-Rasmussen ( $\square)$

Department of Medical Endocrinology PE 2132, National

University Hospital, Rigshospitalet, Blegdamsvej 9,

2100 Copenhagen, Denmark

e-mail: ufeldt@rh.dk
In 2011, the ATA guidelines [2] mentioned that subclinical hypothyroidism has been associated with adverse maternal and fetal outcomes, and recommended that women with positive antibodies against thyroid peroxidase or thyroglobulin and subclinical hypothyroidism should be treated with LT4. The same guidelines also recommended LT4 replacement in women with antibody negative subclinical hypothyroidism, although the evidence levels were very low both for obstetrical and neonatal neurological outcome. The current study [1] does not mention any measurement of thyroid antibodies, and the etiology of subclinical hypothyroidism is, therefore, quite unclear; the study does not include an untreated control group, which would have been advantageous for comparison of the biochemical evolution of the thyroid function tests, considering in particular that serum TSH is very often lowered in early pregnancy due to an effect from human chorionic gonadotropin [3]; the study does mention the use of trimester-specific reference concentrations for serum TSH and free T4, but do not take into account the high degree of intraindividual variation of the concentrations, even in pregnancy [4]; the strict cut-off concentrations necessary for conducting the study are very dependent on method and laboratory [5], which should have been discussed in the paper. Even though the study mentions cut-off concentrations for TSH and free T4, the authors do not mention, how many were outside target in each method, which would have been useful information for the clinician.

One of the important points for discussion within this topic has been whether to screen all pregnant women for thyroid dysfunction and autoimmunity, but up to now guidelines have advocated for case-finding although more groups of women with predisposition for hypothyroidism are included in the new guidelines [2]. The presented study does not add to this discussion, since the data are not suitable for answering this question. The study can only 
answer what to expect concerning development of thyroid function tests if you choose to treat women with different concentrations of serum TSH. That does not, however, indicate whether or not the treatment was beneficial since no control group was included and no outcome variables of pregnancy or neurocognitive development of the baby were included [6, 7].

The study, therefore, does not aid in answering the question whether or not women with subclinical hypothyroidism and no autoantibodies should be treated at all. However, if the clinician chooses to replace with LT4 in pregnant women with subclinical hypothyroidism by virtue of the potential benefits outweighing the potential risks as recommended by the international task force, the treatment should be monitored closely by monthly measurements of thyroid function, and treatment adjusted accordingly in order to maintain normal biochemical variables. Serum TSH-related fixed doses provided a pragmatic way of commencing therapy rather than a single fixed dose [8]. With these limitations in mind the study has added a small piece to the continuous puzzle of how to manage subclinical hypothyroidism in pregnancy.

\section{References}

1. X. Yu, Y. Chen, Z. Shan, W. Teng, C. Li, W. Zhou, B. Gao, T. Shang, J. Zhou, B. Ding, Y. Ma, Y. Wu, Q. Liu, H. Xu, W. Liu, J. Li, W. Wang, Y. Li, C. Fan, H. Wang, H. Zhang, R. Guo, The pattern of thyroid function of subclinical hypothyroid women with levothyroxine treatment during pregnancy. Endocrine (2013)

2. A. Stagnaro-Green, M. Abalovich, E. Alexander, F. Azizi, J. Mestman, R. Negro, A. Nixon, E.N. Pearce, O.P. Soldin, S. Sullivan, W. Wiersinga, American thyroid association taskforce on thyroid disease during pregnancy and postpartum. Guidelines of the American thyroid association for the diagnosis and management of thyroid disease during pregnancy and postpartum. Thyroid 21, 1081-1125 (2011)

3. U. Feldt-Rasmussen, E.R. Mathiesen, Endocrine disorders in pregnancy: physiological and hormonal aspects of pregnancy. Best Pract. Res. Clin. Endocrinol. Metab. 25, 875-884 (2011)

4. M. Boas, J.L. Forman, A. Juul, U. Feldt-Rasmussen, N.E. Skakkebaek, L. Hilsted, M. Chellakooty, T. Larsen, J.F. Larsen, J.H. Petersen, K.M. Main, Narrow intra-individual variation of maternal thyroid function in pregnancy based on a longitudinal study on 132 women. Eur. J. Endocrinol. 161, 903-910 (2009)

5. U. Feldt-Rasmussen, A.S. Bliddal Mortensen, A.K. Rasmussen, M. Boas, L. Hilsted, K. Main, Challenges in interpretation of thyroid function tests in pregnant women with autoimmune thyroid disease. J Thyroid Res. 2011, 598712 (2011). doi:10.4061/2011/ 598712

6. B.M. Casey, J.S. Dashe, C.E. Wells, D.D. McIntire, W. Byrd, K.J. Leveno, Cunningham FG subclinical hypothyroidism and pregnancy outcomes. Obstet. Gynecol. 105, 239-245 (2005)

7. V.J. Pop, E.P. Brouwers, H.L. Vader, T. Vulsma, A.L. van Baar, J.J. de Vijlder, Maternal hypothyroxinaemia during early pregnancy and subsequent child development: a 3 years follow-up study. Clin. Endocrinol. (Oxf.) 59, 282-288 (2003)

8. I. Seoane Cruz, M. Penı'nA' lvarez, R. Luna Cano, R.V. Garci'aMayor, Treatment with fixed thyroxine doses in pregnant women with subclinical hypothyroidism. Endocrinol. Nutr. 59, 284-287 (2012) 